\title{
Educate to Keep It Great: The Long-Term Successes of a Virtual Great Barrier Reef Education Program
}

\author{
P.F. Francis', J. Spencer², and C. McGrogan² \\ ${ }^{1}$ School of Life and Environmental Sciences, Deakin University, Waurn Ponds Campus, Geelong, Australia and ${ }^{2}$ Great Barrier Reef Marine Park Authority, Reef HQ \\ Aquarium, Townsville, Australia \\ Keywords: Community Outreach, Distance Education, Environmental Education, Great Barrier Reef, Virtual Field Trip \\ Publication Date: August 10, 2020
}

DOI: https://doi.org/10.15695/jstem/v3i2.03

\begin{abstract}
The Great Barrier Reef (GBR) is under pressure from climate change and other threats, and part of the solution is to raise awareness of the GBR and its threats beyond a local audience (Great Barrier Reef Marine Park Authority, 2019). Reef HQ Aquarium (the national education centre for the Great Barrier Reef Marine Park Authority) implemented a virtual marine education program focussed on the GBR in 1998. The program uses videoconferencing technology to connect real-time marine biology experts to participants around the world. We report on $>$ ten years of participant trends for this program to assess whether the program is a mechanism for increasing community educational outreach. Since 2005, the program increased the Aquarium's community and educational outreach by $4.8 \%$ by connecting an additional 83388 participants from 19 countries worldwide. While the videoconference technology has evolved overtime, the program content remains a unique, interactive virtual experience that has proven to be an effective way for an informal learning institute to increase their outreach on a national and international level, particularly as there has been a transition to online learning and educational programs due the recent global Coronavirus pandemic.
\end{abstract}

\section{INTRODUCTION}

Today, distance or outreach education using interactive videoconferencing technology is used on a regular basis following the improvements of technology and internet accessibility. Classrooms are connecting to informal learning institutes such as aquariums or museums, to engage with an expert on the topic covered in class. This type of connection is commonly referred to as a virtual field trip (VFT). VFTs are a great solution for student cohorts to visit places that might not otherwise be possible due to location, time, cost or risk associated with a physical field trip (PFT) (Majherová et al., 2014; Placing and Fernandez, 2002; Spaulding and Ranney, 2008). For example, students in Alaska can connect to marine biologists on the Great Barrier Reef in Australia or Australian students can interact with experts at the Johnson Space Center in America. Both these examples would generally not be achievable via a PFT, as cost, time and location would play major hurdles in achieving this.

As we now face a new learner, the use of information and communications technology (ICT) in the classroom is commonplace and more educators around the world are adopting VFTs as a regular component to their curriculum (Lawson et al., 2010). Generally, teachers find VFTs to be a flexible learning tool (Norris et al., 2015), and VFTs have been documented to enhance and improve science education (McCombs et al., 2007). The benefits for students learning through VFTs have been shown to increase their motivation and their engagement with the content due to the connection to the real world (McCombs et al., 2007; Tuthill and Klemm, 2002).

Given the benefits to VFTs, there is still resistance based on several factors: lack of ICT training in this area, time to incorporate VFTs in an already heavily prescribed curriculum, and student's concerns of VFTs replacing PFTs (Norris et al., 2015; Spaulding and Ranney, 2008; Spicer and Stratford, 2001). The solution may be to offer a blended approach; where VFT could be used as a precursor for PFTs or alternatively, used as a revision tool post PFT (Spaulding and Ranney, 2008; Spicer and Stratford, 2001). This blended 
method has been adopted in classrooms before where enhanced knowledge was shown by students who participated in this combined learning experience (VFTs and PFTs) compared to students who only participated in a learning experience with just a PFT or a VFT (Majherová et al., 2014). A specific example of this blended approach is a school based in Western Australia using a coral reef VFT experience (e.g. Great Barrier Reef virtual connection with Reef HQ Aquarium in Queensland) prior to the class visiting a coral reef (PFT) closer to their physical location (e.g. Ningaloo Reef, Western Australia).

Not only are there benefits for the school community, VFTs have allowed informal learning institutions (e.g. museums, aquariums) and professionals working in various fields of industry (e.g. scientists) to share their expert knowledge and achieve their organization's educational outcomes by interacting with students in a real-time environment (Dees, 2008). Referred to as content providers, informal learning institutions can potentially expand their community and educational outreach range via this method (see Dees, 2008 for an example). There are now hundreds of worldwide videoconferencing content providers that are listed on reputable online, searchable databases of distance learning program offering websites where schools can book their VFTs (website examples include: Center for Interactive Learning and Collaboration (CILC), https://www.cilc.org; in the USA or Distance and Rural Technologies Connections (DART), https://dartconnections.org.au in Australia). A content provider listed on both these sites is Reef HQ Aquarium, located in Townsville, Queensland, Australia. Reef HQ Aquarium provides a one of a kind VFT program delivering education content relating to the Great Barrier Reef (GBR) delivered live by a scuba diver.

The GBR is a Marine Park and a World Heritage Listed site that attracts millions of visitors to Queensland in Australia every year. At approximately $2300 \mathrm{~km}$ long, the GBR makes up $10 \%$ of the world's coral reef ecosystems (Great Barrier Reef Marine Park Authority, 2018). The biodiversity on the GBR is diverse with 600 types of soft and hard corals, 3000 species of mollusks, 1625 species of fish, 133 types of sharks and rays and at least 30 species of whales and dolphins (Great Barrier Reef Marine Park Authority, 2018). Being one of the most biologically diverse ecosystems on earth, the GBR is a popular topic integrated into the science and geography learning areas of the Australian Curriculum. For example, "Scootle" which is an online digital resource site for Australian teachers, provides the resource "Biodiversity and the Great Barrier Reef teacher resource booklet" which directly aligns with the Australian Curriculum. However, with the increasing levels of environmental threats facing the GBR, across multiple scales (local, regional and global) educating the wider community beyond the Australian classroom about these threats has become increasingly important for the primary Agency responsible for managing the GBR; the Great Barrier Reef Marine Park Authority (the Authority).

One of the many ways the Authority achieves this is via their national education center - Reef HQ Aquarium. Located in Townsville, Queensland, the Aquarium offers visitors living exhibits that showcase the diversity of the GBR complemented by interactive educational experiences to raise awareness of the threats to the GBR and in turn, encourages people to adopt positive environmental behavioral changes. Visitation to the Aquarium averages 138,862 visitors per annum (average calculated on 31 years of data), consisting of local, national and international visitors. However, Reef HQ Aquarium looked beyond this localized approach and in 1998 implemented a virtual reef education program to regional schools in Queensland, Australia. Today, the program reaches a worldwide audience where participants can experience the wonders of the GBR through connecting and interacting in real-time with a qualified marine biologist via state-of-the-art videoconferencing technology. This paper reports a 14-year trend of participants in the Reef Videoconference program to assess whether it has been a viable mechanism to increase outreach and deliver its core mission of raising awareness and encouraging behavioral changes not only on a local and national scale, but also encouraging changes at the international level.

\section{METHODS}

The Reef Videoconferencing Outreach Program: Evolution of Reef Videoconferencing. Reef HQ Aquarium opened in 1987 with the mission to educate and inspire visitors to care for the future of the GBR. As part of achieving this mission, Reef HQ Aquarium offers daily talks and tours to the general community as well as curriculum-based education programs to early childhood, school and tertiary groups. While most of the curriculum-based programs are delivered to students physically visiting the site, there was a growing need to expand the outreach beyond the GBR shores and take the program to a wider audience. The creation of "Reef Videoconferencing" was first developed in 1998 following funding from Telstra IT Learning Project. The program began with a partnership between Reef HQ Aquarium, Videolink Taskforce (TAFE Queensland), Barrier Reef Institute of TAFE and Education Queensland. The initial project saw Queensland schools partake in 3-5 week long modules focusing on marine ecology and management. In 1999, the initial funding for the program ceased and it was not until 2003, Sony sponsored the program by delivering a new videoconferencing system (Sony PCS-1P) that enabled multisite capabilities. The technology upgrade was perfectly timed with the release of the blockbuster movie "Finding Nemo" where the GBR was showcased to audiences around 


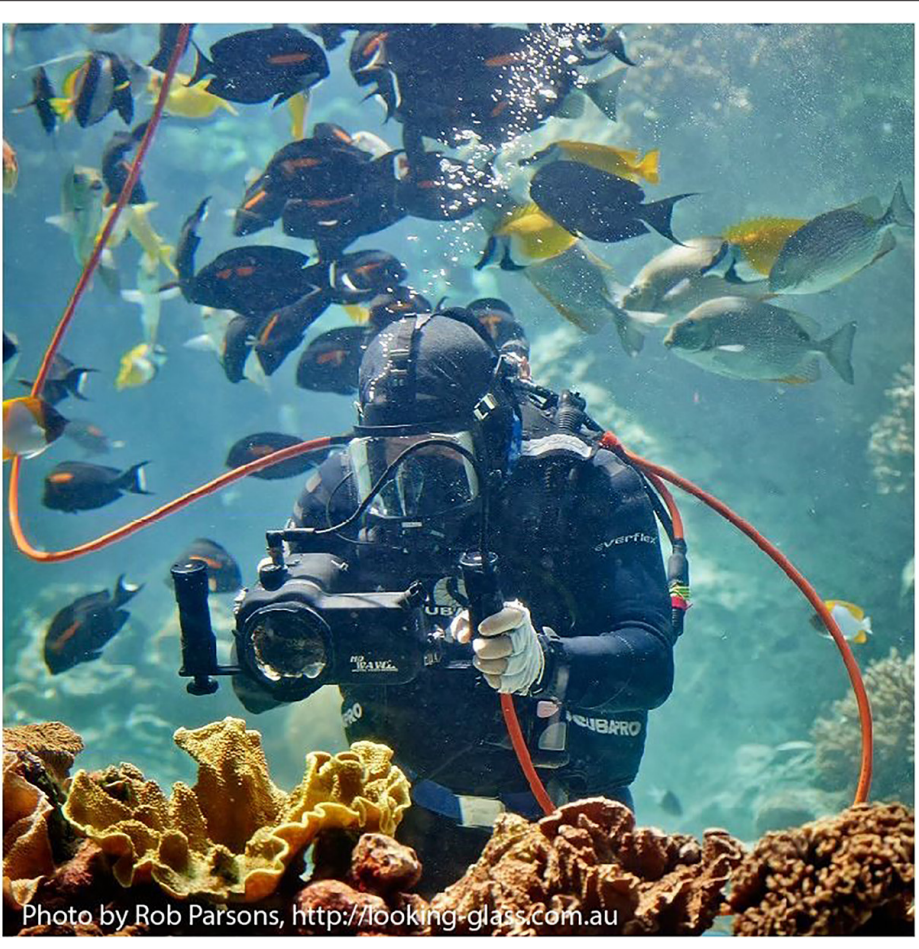

Figure 1. One of the Marine Education Officers presenting the Reef Videoconferencing program underwater in the 2.5 million-liter coral reef exhibit at Reef HQ Aquarium. Photo credit: Rob Parsons, http://looking-glass.com.au

the world and with it, reignited a passion for the reef and a drive to know more. After the Sony sponsorship ended in 2008, a new partnership between Poly and Reef HQ Aquarium was established. The program technology was upgraded in 2008 with Polycom ${ }^{\circledR}$ RealPresence ${ }^{\mathrm{TM}}$ Room solution, HDX ${ }^{\circledR} 8000$. Reef HQ Aquarium became a content provider for Poly and with this, an opening into the American and Canadian education sector and therefore creating a long-term sustainability of the program.

In 2015, there was an emergence of a number of cloudbased virtual meeting room platforms, offering content providers easier connectivity with participants. Whereas previously connections were restricted to the availability of dedicated H.323 videoconferencing technology, the cloudbased meeting platform allowed participants to connect to Reef Videoconferencing through a laptop, PC or other similar smart devices. As the Zoom platform appeared to be growing in popularity ahead of others, Reef HQ Aquarium began trials with it in October 2015, before adopting it as an option for participants in November 2015. Since then, Reef HQ Aquarium has delivered 65 connections via Zoom, compared to 200 using dedicated videoconferencing technology. The authors predict that online cloud-based communication applications (e.g. Zoom, Microsoft Teams, Google Hangouts) will become the preferred platform for education institutions connecting to VFTs, particularly as these applications have been a regular communication platform between school teachers and students during recent times when schools have been closed in response to social distancing restrictions enforced due to the global Coronavirus pandemic in 2020 .

Program Format. The Reef Videoconference virtual excursion connects the audience to the GBR and does this by interacting with a marine educator while they are SCUBA diving in one of the two largest living exhibits on site at Reef HQ Aquarium, Townsville (see Figure 1). The program begins in the 2.5 million-liter coral reef exhibit (the world's largest living coral reef aquarium) where students are taken on a virtual dive to learn about how corals help build the reef and some of the charismatic animals that call the reef home; all the while discussing the environmental issues concerning the GBR. Students are given many opportunities to interact with the presenters and ask any questions they may have about the GBR. The second half of the program is conducted in the 750,000-liter predator exhibit where students see close up vision of a variety of species of sharks and rays and the larger predatory fishes of the GBR. The program usually runs for one hour and as a minimum requires three Reef HQ Aquarium staff members. Reef HQ Aquarium is a non-forprofit organization and as such, the fee required to partake in the program covers the running costs of the program (e.g. technology, salaries and equipment). The fee (one-hour program; AUD \$350) has not changed in seven years, ensuring returning participants and maintaining a high standard of education which has been recognized by receiving prestigious content provider awards (see Discussion for award details). The program is advertised on at least two searchable databases of distance learning program offering websites (national and international based websites - DART and CILC) where schools can book their virtual programs. Reef HQ Aquarium also advertises via its own website (see https:// www.reefhq.com.au/education/Virtual-Connections). Once Reef HQ Aquarium receives a booking notification (via their education email account), the education team will communicate with the client via email where the program content is discussed and, if requested, how the content can be tailored to the audience or the unit of work being studied. A test connection is then performed prior to the 'live' VFT. This is usually done at least several days prior to allow time for any technology issues to be resolved if needed. During the test connection, the format of the presentation and any finer details of the content are also discussed. Following each VFT, a follow up email from an education team member is sent to the client thanking them for connecting for a VFT, and more recently (since 2018) encouraging the client to complete an online feedback survey (via Survey Monkey).

While there is no requirement for students to undertake pre-study prior to the VFT, some teachers choose to get their students to prepare by getting them to write down their questions about coral reefs or the GBR beforehand. In some cases, these questions are collated by the teacher and sent 


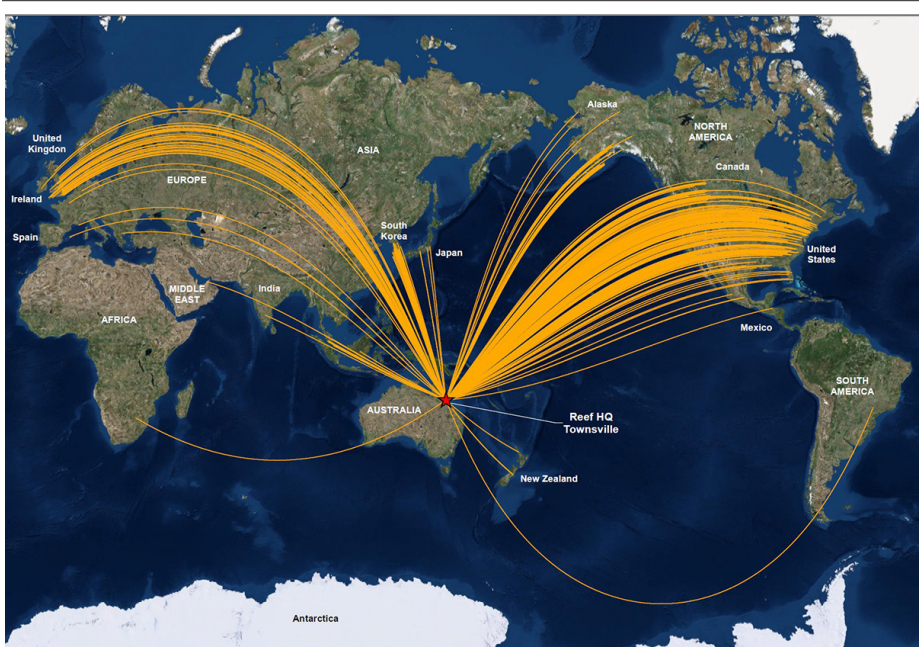

Figure 2. World map displaying the outreach of the Reef Videoconference program conducted by Reef HQ Aquarium (red star = Townsville, Australia) from 2005 - 2018. Each solid, orange line depicts a location where the Reef Videoconference program has connected on a worldwide basis.

to Reef HQ Aquarium staff in advance so that they can be answered during the VFT. In other cases, students are chosen to read their pre-prepared questions out to the presenter during the session. However, the most common approach adopted is presenters answering questions on the spot while participants are connected in the VFT. The authors believe that this flexible approach to answering questions during the program adds to the level of student engagement and is one of the contributing success factors (see Discussion).

\section{RESULTS}

Outreach Participants Trend. The Reef Videoconferencing virtual outreach program has conducted 1,300 Reef Videoconferences to 19 different countries (see Figure 2) and connected with 83,388 participants since 2005 . Connections within Australia were the most frequent (678 out of a total of 1,252 connections from 2005 - 2018; Figure 3) followed by America (362 out of a total of 1300 connections from 2005 -2018; Figure 2). The high trends for Australia and America are most likely contributed to Reef HQ Aquarium being a registered content provider on two booking websites located in both the two countries (e.g. DART - Australia and CILC - America). Additionally, the program is restricted to daylight hours (between 8am and 4pm) to optimize light for the underwater video connection. This restriction can conflict with time differences between Australia and other countries where in some instances; the connections need to be made outside of normal school hours.

Most of the connections within Australia were focused into New South Wales (NSW) classrooms (71\% of the total Australian connections were to schools within the state

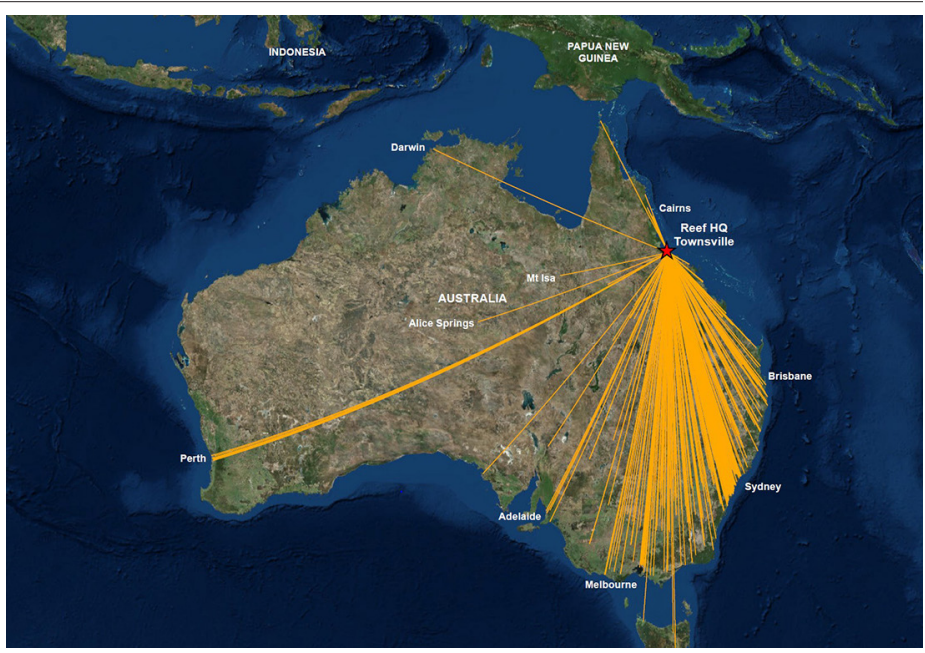

Figure 3. Map of Australia showing the Reef Videoconferencing connections made across Australia from Reef HQ Aquarium, Townsville (red star) during 2005 - 2018. Reef HQ Aquarium is located in Townsville, Australia (red star $=$ Townsville). The solid, orange lines extending beyond the red star show the locations to where the Reef Videoconference has been delivered.

of NSW; Figure 3). This high percentage is related to the relationship between Reef HQ Aquarium and the DART Connections unit of the New South Wales Department of Education and Communities. DART Connections provide and coordinate videoconference excursions to schools and TAFEs within NSW after the NSW government's expansion of videoconferencing technology to every school and TAFE campus in NSW. DART Connections provide a marketable platform for content providers listed on their site across all NSW state schools. Schools residing in other Australian states and territories usually book their program directly with Reef HQ Aquarium.

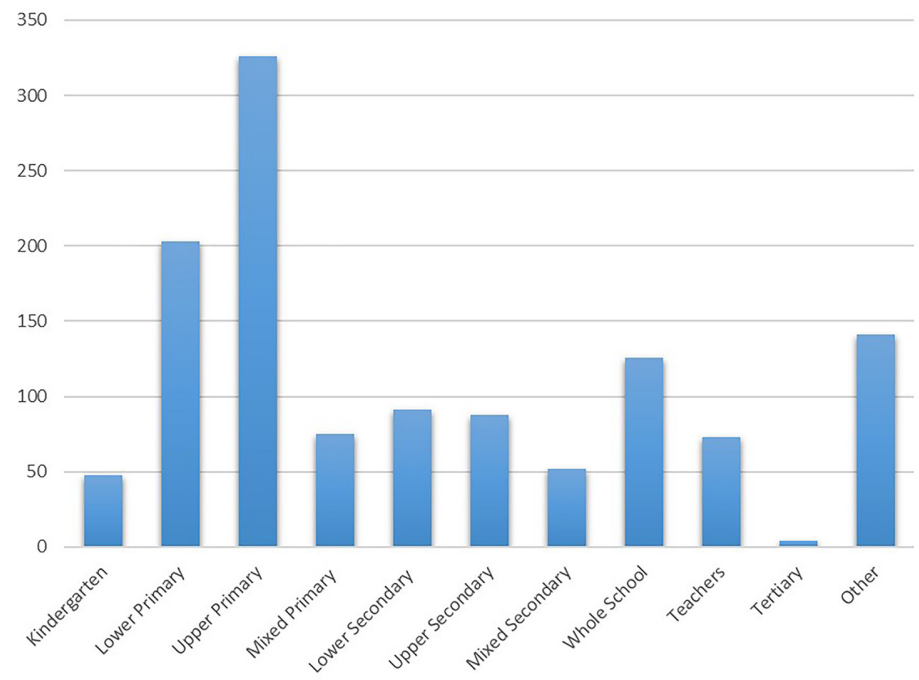

Figure 4. Total number of Reef Videoconference participants grouped by school levels (x-axis; mean data from 2005-2018). Other $=$ participants were not school specific . 


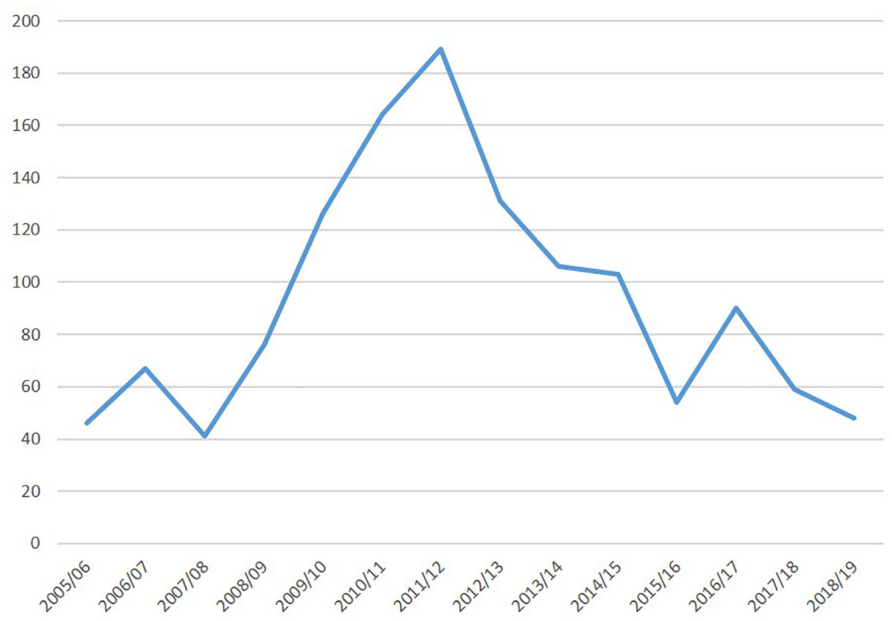

Figure 5. Total number per financial year of Reef Videoconferences delivered by Reef HQ Aquarium from 2005 - 2018.

Over the 14-year period, primary school students were the most common participants in the program with upper primary school students (Grade $4-6$; usually $10-12$ years old) connecting the most (326 connections; Figure 4). The Reef Videoconferencing program is ideal for primary school aged children as it complements the biological science understanding of the Australian Curriculum across these year levels (e.g. Year 4; Living things depend on each other and the environment to survive ACSSU073, Year 5; Living things have structural features and adaptations that help them to survive their environment ACSSU043, and Year 6; The growth and survival of living things are affected by physical conditions of their environment ACSSU094; Australian Curriculum, Assessment and Reporting Authority 2019). While VFTs are also conducted to high school groups, these are of much smaller number and are usually limited to those students studying geography subjects or senior marine science.

An interesting trend to note occurred during the 2011-12 financial year where the highest number of videoconferences were delivered (189; Figure 5). The spike of connections was due to Reef HQ Aquarium in collaboration with the Australian Government's Department of Foreign Affairs and Trade, participating in the global trade event Yeosu Expo in Korea. Their participation in the Expo led to 97 daily live video connections from the Australian Pavilion at the Expo to Reef HQ Aquarium. The theme of the Expo was 'The Living Ocean and Coast' and the connection to Reef HQ Aquarium was to not only promote Australia as a tourist destination, but to also showcase Australia's strong research and environmental capabilities. The partnership led to another collaboration between Reef HQ Aquarium, and the Department of Foreign Affairs and Trade where a further 30 Reef Videoconferences were conducted with Korean schools. These were also supported by collaboration with the University of New England, which connected schools in NSW and Korea

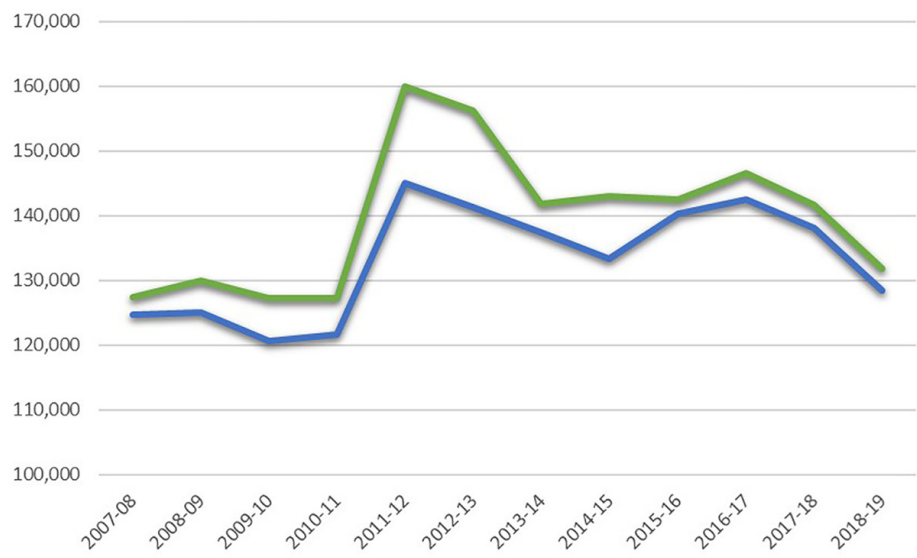

Figure 6. Total visitation numbers per financial year to Reef HQ Aquarium from 2007 - 2018 (excluding the Reef Videoconferencing program; blue line) and total visitation rate per financial year with the inclusion of the Reef Videoconferencing program (green line).

to jointly participate in the reef video conference sessions. These additional connections contributed to the high amount of Reef Videoconferences seen in the 2012-13 financial year (131 connections; Figure 5).

\section{DISCUSSION}

Virtual Outreach Success. The addition of the Reef Videoconference to the education programs at Reef HQ Aquarium has not only increased visitation rates (even if indirect) to the Aquarium but has also widened the educational outreach beyond Townsville. Since 2007, the Aquarium education team has delivered their education programs to 146,646 students. Interestingly, $53 \%$ of students were connecting virtually via the Reef Videoconference program. Furthermore, the implementation of a virtual education program since 2007 has increased the audience outreach of the Aquarium on average by $4.8 \%$ (see Figure 6 ). Since 2005, the program has had 744 different groups (e.g. school, university, conferences, etc.) connect, and from this, 197 groups have elected to connect again, with 1 group connecting 12 times over the time frame. A return rate of $26.5 \%$ ensures the program continues each year, but also highlights that this program is predominately servicing singular connections while still maintaining the longevity of the program.

Schools that book VFT programs via the CILC website are invited via an online notification to complete a post-evaluation of the program they have participated in. The survey evaluates the program (1. engagement level; 2. age appropriate; 3. alignment to Education Standards; 4. supported curriculum and instruction goals) and the presenter(s) (5. presenter's knowledge on content delivered, 6. good rapport established with the participants and 7. presenter's engagement). Over the same period as the previous long-term connection data (2011-2018), Reef HQ Aquarium received 


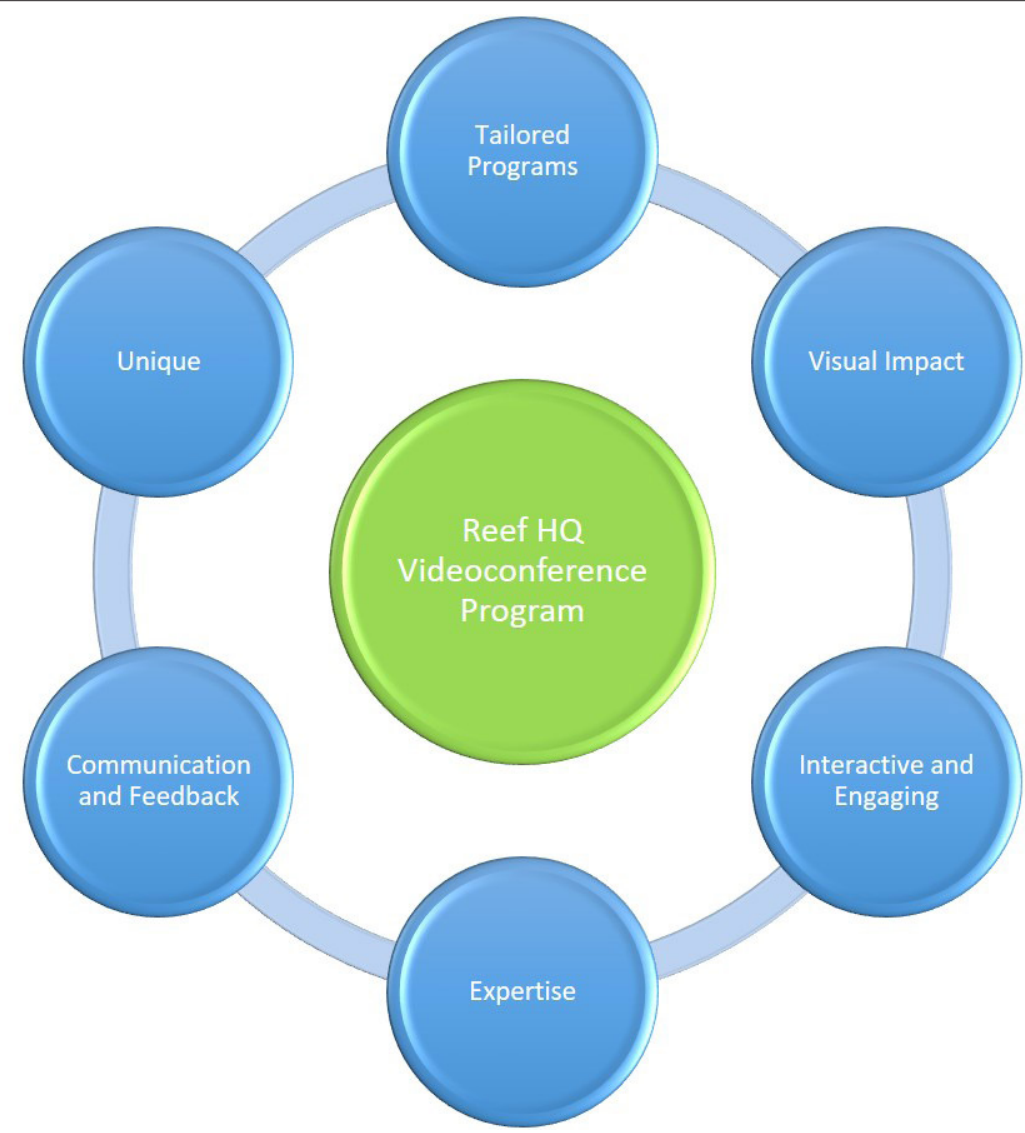

Figure 7. The circular framework of factors that are implemented together that contributes to the long-term success of the Reef Videoconference program at Reef HQ Aquarium.

a subset of survey evaluations from 45 schools, which had voluntarily responded to the CILC surveys post connection. This represents $12.4 \%$ of the total CILC schools participating in Reef HQ Aquarium's VFTs during this timeframe and thus, cautionary analysis of the feedback data needs to be considered. From the 45 groups surveyed, 84\% strongly agreed the presenters were interesting and engaging, 100\% strongly agreed the presenters were knowledgeable and 98\% of groups strongly agreed the presenters established a good rapport with participants (Likert scale options: strongly agree, agree, disagree, strongly disagree or not applicable). Concerning the program content, the 45 groups surveyed strongly agreed that the program supported the curriculum $(87 \%)$ and educational standards $(78 \%)$ and the program content was engaging (82\%) and appropriate for the participants age and abilities (87\%). While this feedback is encouraging and valuable to measure the program's impact and identify areas of improvement, we also recognize this is a small sample size and an area for future direction. DART Connections also collect feedback from schools; however, this is often in relation to the quality and success of the connection. The metrics used are different to those of CILC and the data sets received by Reef HQ Aquarium were ad hoc and therefore difficult to quantify and report here.

The evaluations that are collected by both CILC (and
DART) are generic surveys, which are generated for all VFT content providers. They are not specific to Reef HQ Aquarium. In recent years, Reef HQ Aquarium identified the need to develop its own specific evaluation survey which also includes questions to ascertain whether the VFT helps to achieve the Authority's educational outcomes. It was also identified that these surveys should capture all groups undertaking a VFT, not just those booked through CILC.

In 2018, Reef HQ Aquarium developed its own evaluation survey, modelled off the format of the CILC evaluation, but also including specific questions including whether participants gained an increased awareness of the threats to the GBR and what actions they can take to help protect it, as a result of the VFT. The education team has recently implemented an invitation to complete the online survey (via Survey Monkey) in all their post-communication correspondence to the participant. This survey is in the early infancy, and as such, data from this survey has not been presented in this paper. We recommend informal institutes who are considering to transition to VFTs, to consider creating an online feedback survey prior to commencing VFTs for their own program analysis, and also to develop mechanisms to encourage participants to complete the surveys and return them.

The Reef Videoconference educational content and pro- 
gram delivery has received consistent outstanding scores by program evaluators. The Pinnacle Award is an annual event where content providers registered through CILC are scored based on the publicly rated survey provided by the participant's feedback on their VFT experience. To be eligible for a Pinnacle Award, content providers must have conducted more than 10 connections in one year with at least three of those connections completing a post connection survey. The survey asks participants to rank using a Likert scale response with regards to the seven ranking criteria outlined above. The Likert scale response is then allocated a scoring system: strongly agree ( 3 points), agree ( 2 points), disagree ( 1 point) and strongly disagree ( 0 points). The total points are then calculated and an average out of 3 obtained. A score above 2.85 receives a Pinnacle Award. Testimonials from past participants completing this post-evaluation survey can be found via following link on the CILC website: https:// www.cilc.org/ContentProvider/ViewContentProvider.aspx?ID $=149$.

The Pinnacle Awards were established in 2008. In the 12 years since the awards have been established, Reef HQ Aquarium has been recognized with 11 Pinnacle Awards and one honorable mention (the awards have been based on 157 evaluations over this timeframe). To the authors knowledge few other organizations have received a Pinnacle Award to this level of consistency over such a long period of time. These awards are reflective of the uniqueness the program brings to the VFT platform and a great benchmark for other content providers when designing a live and interactive VFT.

Quantitatively, the Reef Videoconference program has proven successful with increasing the Aquarium's outreach goals and delivering a program that satisfies the client's educational needs. The authors have identified a framework of six factors they believe contribute to the long-term success of this program (see Figure 7); with some of the factors taken from best practices recommended in Greenberg and Colbert (2003) and Peterson (2000). The factors; tailored programs, visual impact, interactive and engaging, expertise, communication and feedback and unique are discussed independently below but each are considered part of an integrated framework that without one factor present, the success of the program could be disrupted.

Tailored Programs. "Introduction to the Great Barrier Reef" is the default Reef Videoconference program delivered, which covers a broad overview of reef biology and ecology such as coral formation, symbiotic relationships, animal feeding behavior, animal reproduction, marine conservation, adaptations and classification. Despite this program being the most popular, the Reef Videoconferences can be specifically tailored to a topic complementary to the curriculum needs of the client. For example, topics such as coral bleaching and other threats to the GBR (including climate change) are popular choices requested for senior secondary Geography units. This ensures the content delivered is relevant to the audience and more likely to enhance the learning outcomes to the students (Peterson, 2000). Furthermore, the education team ensure each connection is personalized for the group. For example, the presenters will often research where the connecting group is located and ensure that they show this personalization embedded in the connection. This personalization is often commented from teacher feedback post-connection:

The personalization and interaction of the program was excellent. The mention of our town park and even an upcoming program we were having was just an amazing touch.

The experts at the aquarium will tailor their presentation according to your goals and grade level. They make it fun and interesting to the point everyone will be thanking you.

Visual Impact. The presenters ensure the audience are exposed to a variety of visual backdrops and presentation techniques, which enhances the overall effectiveness of the presentation and maintains the attention of the participants (Peterson, 2000). Participants will view footage from the coral reef and predator tanks, view images and content via green screen technology and talk face-to-face with the two presenters. The SCUBA presenter can direct footage dependent on the Aquarium's conditions and the participant's requirements. For example, visual footage could be the SCUBA diver talking to the audience underwater or viewers watching a large school of herbivorous fish eating their lunch or the presenter zooming up close to a coral colony to see the individual polyps. Steps are also taken to improve the overall effectiveness of the presentations based upon attributes described in Peterson's (2000) guide for videoconferencing. The Reef Videoconference program considers overall effectiveness by adopting the following: a) the underwater presenter ensuring a slow, natural movement of the camera, b) presenters wearing clothing/wetsuits in solid colors and, c) text used in the shared content is large enough for reading.

Interactive and Engaging. The most common feedback from VFT audiences is to not only deliver excellent content, but to also keep the program as interactive as possible to maintain engagement (Greenberg and Colbert, 2003; Peterson, 2000). This is something the Reef Videoconference program excels at with interactive dialogue, asking and answering questions throughout the program and encouraging participants to use hand gestures (e.g. students are taught dive hand signals at the start of the program and are then encouraged to use them throughout the program to communicate with the SCUBA diver). The presenters will also use 
other interactive games and physical movement throughout the program if they feel the engagement is wavering. Reef Videoconferencing program often receives feedback from teachers regarding the inclusion of student questions and interactivity with the presenter. Below are a small sample of teacher feedback regarding this:

The students were engaged the entire time and were able to have questions answered and shown!

Taking time to answer questions no matter how small, was a priority that everyone appreciated.

I love the interaction and the fact that diver was able to show students (and myself) up close and personal the underwater world of the reef.

The instructors were flexible enough to answer questions and return to particular subjects if the students wanted to learn more.

This is not a watch, listen and learn program. It is interactive throughout and guided by student questions. Excellent program!

Expertise. One of the motivating reasons classroom teachers seek VFTs programs is so the students have the opportunity to connect and interact with an expert on the topic of interest (Spaulding and Ranney, 2008). Reef HQ Aquarium have a dedicated education team who deliver the education programs offered at the local, face-to-face delivery method and the virtual programs. Each member of the team, has expertise in either education or marine biology (or both) and are well versed with topics surrounding the Great Barrier Reef.

Prior to presenting, each new team member is trained in every program and are required to meet the standard of marine interpretation delivered at the Aquarium. This ensures each team member are subject matter experts and can answer questions relating to any specific content about the Great Barrier Reef and ensure the answers are tailored to the targeted audience (this is one of the signs of spectacular content recommended by Greenberg and Colbert, 2003). Delivering a VFT program at Reef HQ Aquarium requires extensive training where staff would have previously demonstrated their knowledge and communication competency via the delivery of the Aquarium's PFT programs. The main challenge for a new staff member is knowledge and confidence to use and troubleshoot the technology required to deliver the VFT. Overcoming this challenge requires repetitive observational learning where the trainee will observe numerous VFTs first and when deemed confident to progress to the next level of training, the trainee will co-present alongside an experienced staff member as a "dry-side" presenter. Staff who are trained to be the SCUBA presenter, require more vigorous training. This training involves having the relevant SCUBA training that adheres to Reef HQ Aquarium's diving policy and then, beginning their on-site training as a dive buddy during numerous daily dives. Once the diver is deemed confident to progress to presenting while SCUBA diving, the trainee will practice their skills with co-presenting in the daily, SCUBA presentations offered to the public visiting the Aquarium. The SCUBA presenter will have also presented a VFT as the "dry-side" presenter prior to their first VFT SCUBA delivery.

This level of training allows the staff member to experience the range of questions asked from the audience and to familiarize themselves with the technology. Staff conducting VFTs have experience already with conducting the daily PFT programs that are offered at Reef HQ Aquarium, and are thus, equipped with extensive knowledge of the Great Barrier Reef ecosystem. There are of course times when staff are unable to answer specific questions during the VFT, but the team endeavor to find the answer via the appropriate channels and then communicate this answer back to the teacher via post connection correspondence.

For VFT's with specialist content requests, the education team will draw upon the expertise of the more senior education staff to be the dry side presenter. At times, they have also invited 'special guest presenters' including other staff members of the Authority with particular subject matter expertise to be co-presenters.

While it is important to ensure subject matter experts and high-level educators are delivering the program, it is also just as important to ensure there are team members who can provide technical support with the distance education technology (Greenberg and Colbert, 2003; Warner, 2008). Reef HQ Aquarium has a dedicated member who not only coordinates the Videoconference program, but also is well versed with the technology and provides training to staff involved in the program. This staff member is also supported by other team members who assist with these elements. All staff are trained in camera use and troubleshooting, when and how to switch camera feeds or integrate other presentation tools into the learning environment (such as green screen technology), SCUBA presenting and the equipment set-up, pack-down and maintenance involved with the underwater technology. Additionally, the Authority provide information technology support officers if the education team is unable to resolve any technical issues. The expert team and level of training each presenter has, ensures minimal occurrences of technical difficulties in the technology and therefore reducing the number of booking cancellations. We recommend other informal education organizations who are in the early stages of transitioning to online tours, to ensure staff delivering these sessions undergo a consistent, quality-controlled training in 
the technology, communication and content involved with the virtual delivery.

Communication and Feedback. The participant feedback survey that CILC provide further collect data on the level of communication before the connection. From the 45 surveys collected and provided to Reef HQ between 2011 - 2018, $96 \%$ of the connections strongly agreed (highest rating) that the program provider was available for communication prior to the program. This high percentage is not surprising given the education team offer an open communication with the clientele. Depending on how the booking is made (whether through CILC, DART Connections or direct contact), the team will make direct contact with the school/client to confirm the day and time of the booking, the topic(s) the client would like to cover and arrange a test connection prior to the program delivery. Time zone differences around the world can be a critical factor connecting to a VFT (Warner, 2008) and this factor is certainly evident for this program as it requires adequate light levels in the aquaria tanks to allow for quality video throughout the connection, and as such, the program is restricted to daylight hours of the Aquarium. Therefore, time zone differences need to be considered when scheduling bookings from other countries outside of Australia and other countries may only have scheduled times to book a session, compared to on-demand bookings.

If the booking has been made through CILC, the participant has the opportunity to provide feedback on the program. Furthermore, the education team often receive informal emails from teachers/supervisors who comment on the valuable experience their students had with the program. In 2018 , the education team has implemented a link to an online feedback survey included in their post-communication correspondence. Quite often, teachers will continue communication with the education team with follow up questions about the content delivered that have either come from the teacher themselves or from the student participants. The education team also receive informal feedback via email correspondence from teachers with regards to video quality, content delivered or requests for further supporting resources. The team is accommodating with this type of feedback and post-connection requests. The most common feedback the team receives is teachers requesting supporting educational resources that students can complete pre-post connection. Providing this level of support to teachers has been in the past, an upon-request delivery because of the accessibility of the resources. However, due to the recent demand for accessible, online educational resources due to the global Coronavirus pandemic forcing many schools to transition to remote online learning, the educational resources are now accessible to external users (e.g. teachers) and can be accessed via the via the Reef HQ Aquarium website (direct link: https:// www.reefhq.com.au/education/teaching-resources).
The education team also records the quality of each connection to ensure a quick resolution of any quality control issues. The level of feedback is an important factor that contributes to the success of this program and has also been noted as one of the signs of spectacular content in Greenberg and Colbert (2003).

Unique. The program is co-delivered in real-time by a marine biologist while SCUBA diving. As far as the authors know, up until very recently (with the addition of a new program by Texas State Aquarium), this type of delivery is unique to Reef HQ Aquarium through the content providers of both CILC and DART Connections websites. Other aquariums offer underwater, live footage but the presenter is not SCUBA diving. This uniqueness adds to the engagement and interactivity level of the audience as they are talking with a GBR expert while surrounded by plants and animals of the GBR, and furthermore, the audience are given the opportunity to learn about the technical aspects of SCUBA diving. Below is a small sample of teacher feedback regarding the uniqueness and impact this program has had on the participants:

It connected my students to a place that is about 10,000 miles away from us. It taught them about different ecosystems and different organisms. It has been great to hear the students continue to talk about the experience.

This experience provided valuable reinforcement of lessons learned in class as a tool to see and experience these things live and 'in-person'. It prompted students to ask more questions and want to learn more even after the unit material was over.

I believe programs like this are invaluable. They expose students to people and places they would ordinarily not have access to. I also believe it helps students understand and grow as global citizens...

...Because of this field trip the kids all want to get SCUBA certified, go to Australia, and study oceans. They are still buzzing about it now!...

\section{CHALLENGES, COVID-19, AND THE FUTURE}

The main challenges in delivering VFTs is ensuring that the internet connectivity/speed from either the client or the Aquarium is sufficient to maintain a high quality audio and video feed. Schools may also have to check with (and get permission from) their ITC department to see if there are any firewall restrictions that may prevent them to connecting to outside the school network, and if required, allow these restrictions to be lifted for when the VFT (and any test 
connections) occur. Teachers also have to feel comfortable with using the equipment required for the connection. To help alleviate these issues, a test connection is conducted with the Reef HQ Aquarium team and the client. It is always recommended that the test is conducted from the physical location and using the same equipment that will be used during the live VFT. At the testing phase usually any issues regarding internet speed/connectivity or other technological issues such as audio from microphones and speakers can be identified and resolved. If an issue is identified, the Reef HQ Aquarium staff will book additional test connections to ensure that everything is running smoothly prior to the live VFT.

On the rare occasion, internet speed from Reef HQ Aquarium can be unreliable which can result in the underwater footage being pixelated or static, affecting the overall engagement of the session. The SCUBA presenter will not be aware of this particular issue when the presentation is in a live format, and hence why the "dry-side" is an essential addition to be the "eyes" of the live connection to communicate this visual to the SCUBA presenter who can then adapt their delivery by reducing the camera movement. Depending on the platform, if the internet connection is unstable from the Aquarium, there may be an option to swap their Internet Protocol (IP) lines or the meeting identification. For example, during a recent VFT using the Zoom platform, it was noticed that the video was pixelating due to poor internet connectivity. It was later identified that there was an issue with that particular IP line in Zoom. As Zoom provides several IP address options to choose from, this issue was resolved by switching the virtual meeting to another IP line with better connectivity. Another issue that the staff have sometimes encountered is difficulty communicating with schools in the USA via email due to the school's firewall restrictions. The authors recommend that at least two methods of communication (e.g. email and phone) are established as a means of alleviating this issue.

During the Coronavirus pandemic, the education team has had to rapidly adapt within a short-time frame to change their mode of delivery of their VFTs. Instead of the usual one-to-one (or point-to-point) connection (e.g. Reef HQ Aquarium and one classroom/school per connection), the education team is now connecting via online communication applications (e.g. Zoom) with numerous participants due to remote learning. For example, instead of having one class of 30 students as a 'single participant', the VFT may now have up to $30+$ participants all individually connected at once via Zoom, where items like the chat function is accessible to each participant and thereby, increasing the chat traffic throughout the connection. While this level of engagement is encouraging, it also requires an additional staff member to assist with the facilitation of the program to manage the administrative side of Zoom (e.g. chat function, raising hands function, muting participants, and admitting participants into the program from the waiting room). This change in delivery mode has created a challenge where younger audience members as participants have been chatting amongst themselves using the chat function or unmuting their microphones when not asked, disrupting the presenter. This has required the Reef HQ Aquarium staff as the session host to enable the controls before the session that only allow chat to occur between the participants and the host only, and disabling participants ability to unmute themselves.

One of the positive aspects that has resulted from the pandemic is a rapid exploration of the video conferencing technology. The education team has recently been delivering programs using the Poly equipment to connect through Zoom, and then live streaming this directly to the Aquarium's Facebook page. These sessions are freely available to anyone in the world connected into Facebook or YouTube. While the participants watching the live stream on the Facebook page are not able to directly talk to the diver, they are able to post questions in the comments which can then be relayed by a staff member watching the live feed to the presenters. Adding the live stream component has allowed Reef HQ Aquarium to increase the audience reach of the session to virtually anyone who has access to the internet. This adaptability has also resulted in the education team diversifying their usual virtual program delivery by the flexibility of conducting virtual education sessions anywhere in the Aquarium. Furthermore, given the current restrictions on travel (particularly international travel), this particular addition to the program increases the outreach potential and allowing people to stay connected with the GBR and to inspire and encourage people to visit the GBR post-pandemic travel restrictions.

Reef HQ Aquarium's Reef Videoconference program has evolved with distance outreach education becoming more accessible for many classrooms with technology such as Zoom. Long-term trends have shown it to be a successful virtual education program that benefits the audience (through satisfactorily meeting their education needs) and the content provider (by increasing their outreach levels by $4.8 \%$ ). While the education team has not noticed an immediate increase in the number of VFTs being delivered as a result of the pandemic, it is expected that teachers will be more familiar with online communication platforms post-pandemic, which will potentially see a result in an increase in more virtual connections in the future. Reef HQ Aquarium are currently delivering their school VFT programs to participants connecting remotely from their home which has allowed more participants to join the session by increasing the accessibility of the program. This current delivery has seen a shift from a pre-pandemic format where quite often international schools with different time zones to Australia would host an after-school event where the Reef HQ Aquarium VFT would take place. This shift in delivery may increase the outreach 
by not only connecting with the students (and their teacher), but also to their extended family members joining the session.

The next step is to assess the effectiveness of the program in relation to curriculum learning outcomes. The authors are interested to test pre- and post-knowledge about ocean literacy relating to the GBR and use this data to gain an insight on the impact this program has on improving ocean literacy in school-aged children. The education team will be looking at methods to better capture and measure the effectiveness of the VFTs with reference to meeting the Authority's mission of raising awareness of the GBR, the threats facing the GBR and inspiring others to help protect the GBR for future generations to enjoy.

\section{ASSOCIATED CONTENT}

Supplemental material mentioned in this article can be found uploaded to the same webpage as this article.

\section{AUTHOR INFORMATION Corresponding Author}

P.F. Francis. 2A Bellarine Highway, Queenscliff, Victoria, 3225, Australia.+61 3 55633026.prue.francis@deakin. edu.au

\section{Author Contributions}

The manuscript was written through contributions of all authors. All authors have given approval to the final version of the manuscript.

\section{ACKNOWLEDGMENTS}

The authors would like to thank the Education Team at Reef HQ Aquarium for their on-going efforts to deliver the Reef Videoconference program. We also acknowledge the Great Barrier Reef Marine Park Authority and Reef HQ Aquarium for providing the long-term data for the paper (Great Barrier Reef Marine Park Authority, 2019). We would also like to acknowledge Poly for their generosity of supplying Reef HQ Aquarium the videoconference technology at various points along the Reef Videoconference journey. Thank you to CILC and DART Connections for listing Reef HQ Aquarium as a content provider on their online databases. The study was exempt from ethical review due to the data being pre-existing and non-identifiable.

\section{FUNDING SOURCE}

This paper was funded by the School of Life and Environmental Sciences, Deakin University.

\section{ABBREVIATIONS}

CILC: Center for Interactive Learning and Collaboration; DART: Distance and Rural Technologies Connections; GBR: Great Barrier Reef; ICT: Information and Communications Technology; NSW: New South Wales; PFT: Physical Field Trip; VFT: Virtual Field Trip.

\section{REFERENCES}

Australian Curriculum, Assessment and Reporting Authority. (2019). Science Understanding. Retrieved from https:// www.australiancurriculum.edu.au/f-10-curriculum/science/

Dees, P. (2008). Making the system work: The content provider and videoconferencing in the K-12 classroom. In D.L. Newman, J. Falco, S. Silverman and P. Barbanel (Eds), Videoconferencing technology in K-12 instruction: Best practices and trends (pp. 52-69). IGI Global.

Great Barrier Reef Marine Park Authority. (2019). Great Barrier Reef Outlook Report 2019, Great Barrier Reef Marine Park Authority, Townsville.

Great Barrier Reef Marine Park Authority. (2018). Reef facts. Retrieved from http://www.gbrmpa.gov.au/the-reef/reef-facts

Great Barrier Reef Marine Park Authority. (2019). Reef HQ Aquarium Video Conference numbers 2005-2019 [unpublished dataset].

Greenberg, A., and Colbert, R. (2003). Best practices in live content acquisition by distance learning organizations. Retrieved from http://spectralink.polycom.com/global/ documents/whitepapers/best_practices_in_live_content acquisition_by_distance_learning_organizations.pdf

Lawson, T., Comber, C., Gage, J., and Cullum-Hanshaw, A. (2010). Images of the future for education? Videoconferencing: a literature review. Technology Pedagogy and Education, 3, 295-314, doi: 10.1080/1475939X.2010.513761.

Majherová, J., Palásthy, H., and Gazdíková, V. (2014, December). Virtual excursion in secondary education. Paper presented at the 12th IEEE International Conference on Emerging eLearning Technologies and Applications (ICETA), Stary Smokovec. Abstract retrieved from doi: 10.1109/ICETA.2014.7107602.

McCombs, G., Ufnar, J., and Shepherd, V. (2007). The Virtual Scientist: connecting university scientists to the K-12 classroom through videoconferencing. Advances in Physiology Education, 31, 62-66, doi:10.1152/advan.00006.2006

Norris, E., Shelton, N., Dunsmuir, S., Duke-Williams, O., and Stamatakis, E. (2015). Teacher and pupil perspectives on the use of Virtual Field Trips as physically active lessons. BMC Research Notes, 8, 719, https://doi.org/10.1186/ s13104-015-1698-3

Peterson, R. (2000). "Real World" Connections through videoconferencing - we're closer than you think! Tech Trends, 44, $5-11$ 
Placing, K., and Fernandez, A. (2002). Virtual experiences for secondary science teaching. Australian Science Teachers Journal, 48, 40-43

Spaulding, D., and Ranney, P. (2008). Virtual Field Trips: Advantages and disadvantages for educators and recommendations for professional development. In D.L. Newman, J. Falco, S. Silverman and P. Barbanel (Eds), Videoconferencing technology in K-12 instruction: Best practices and trends (pp. 191-199). IGI Global.

Spicer, J., and Stratford, J. (2001). Student perceptions of a virtual field trip to replace a real field trip. Journal of Computer Assisted Learning, 17, 345-354. doi:10.1046/j.02664909.2001.00191.x

Tuthill, G., and Klemm, E. (2002). Virtual Field Trips: alternatives to actual field trips. International Journal of Instructional Media, 29, 453-468

Warner, F. (2008). The Journey into Distance Learning: Test drives, roadblocks, and destinations. In D.L. Newman, J. Falco, S. Silverman and P. Barbanel (Eds), Videoconferencing technology in K-12 instruction: Best practices and trends (pp. 35-51). IGI Global 